University of South Carolina

Scholar Commons

$11-1992$

\title{
Unobserved Ability, Efficiency Wages, and Interindustry Wage Differentials
}

McKinley L. Blackburn

David Neumark

Follow this and additional works at: https://scholarcommons.sc.edu/econ_facpub

Part of the Business Commons

Publication Info

Published in Quarterly Journal of Economics, Volume 107, Issue 4, 1992, pages 1421-1435.

http://qje.oxfordjournals.org/

(c) 1992 by Massachusetts Institute of Technology Press (MIT Press)

This Article is brought to you by the Economics Department at Scholar Commons. It has been accepted for inclusion in Faculty Publications by an authorized administrator of Scholar Commons. For more information, please contact digres@mailbox.sc.edu. 


\title{
UNOBSERVED ABILITY, EFFICIENCY WAGES, AND INTERINDUSTRY WAGE DIFFERENTIALS*
}

\author{
McKinley Blackburn and David Neumark
}

\section{INTRODUCTION}

An important area of research on the empirical validity of efficiency wage theory has focused on the role of industry effects in explaining variation in wages across workers The persistence of industry wage differentials across time and countries [Krueger and Summers, 1987], and in wage regressions including a multitude of controls [Dickens and Katz, 1987a, Krueger and Summers, 1988], has been interpreted as evidence consistent with efficiency wage explanations of industry wage differentials, and inconsistent with competitive-market explanations (e g , Dickens and Katz [1987b], Katz [1986], and Krueger and Summers [1987, 1988]) This persistence of industry wage differentials complements what may be interpreted as more direct evidence, including, for example, a negative correlation of quit rates with industry wage premiums [Krueger and Summers, 1987], and a positive correlation between product market power and industry wage premiums [Dickens and Katz, 1987a]

One competitive-market explanation of interindustry wage differentials that is not challenged by the persistence of these differentials is that they are due to differences across workers in "unobserved" ablity or quality (unobserved to the researcher but not to the worker or firm) (e g, Murphy and Topel [1987b]) Previous research has attempted to remove unobserved ablity bias in estımated industry effects by estımating first-difference specifications of wage equations, which difference out individual fixed effects [Gibbons and Katz, 1992, Krueger and Summers, 1988, Murphy and Topel, 1987a, 1987b]

In contrast, this paper explores the unobserved abilty hypothesis by using test scores as error-ridden indicators of ability, and family background variables as instruments This approach avolds two potential problems with using first-difference methods to remove omitted-ability bias from wage equation estımates of

\footnotetext{
*We are grateful to Linda Bell, Alan Krueger, David Levine, an anonymous referee, and semmar participants at Clemson University, the Federal Reserve Board, George Washington University, and the Unıversity of California at Berkeley for helpful comments We thank Eugene Wan for programming and research assistance
}

1992 by the President and Fellows of Harvard College and the Massachusetts Institute of 
industry effects the exacerbation of measurement error from misclassification of industry, and selectivity with respect to industry changes ${ }^{1}$ However, this approach may introduce other problems, for two reasons First, it depends on using test scores that are correlated with the type of ability that is rewarded in labor markets Second, because the test scores are undoubtedly errorridden measures of ability, identifying assumptions are needed to correct for measurement error Thus, the approach taken in this paper should be viewed as complementary to first-difference methods The results indicate that ability can account for only a small portion of interindustry (or interoccupation) wage differentials in cross-section wage regressions

\section{Incorporating AbiLity Measures}

Unobserved worker quality or ablity is modeled as a latent or unobserved variable, with intelligence test scores serving as errorridden indicators of this unobserved variable Family background variables are used as instruments for the test scores, to correct for measurement error This general approach mimics that used in the extensive literature on correcting for omitted variable bias in estimating the returns to schooling [Griliches and Mason, 1972, Corcoran, Jencks, and Olneck, 1976, Chamberlain, 1977, Grılıches, 1977, Hauser and Daymont, 1977, Taubman, 1977] Because many of the conditions (such as monitoring difficulties or turnover costs) that might cause the profitability of payng above-marketclearing wages to vary across industries may also vary across occupations, we consider the impact of incorporating test scores on both industry and occupation wage differentials

The wage equation is assumed to be

$$
w=X \beta+D \gamma+\gamma_{A} A+\epsilon,
$$

where $w$ is the logarithm of the wage, $X$ is a vector of human capital variables or other observable measures of labor quality, $D$ is a vector of industry and occupation dummy variables, $A$ is unobserved ability (usually assumed to be fixed over time for an indıvidual), $\epsilon$ is a randomly distributed error, and $\beta, \gamma$, and $\gamma_{A}$ are

1 Grbbons and Katz [1992], Krueger and Summers [1988], and Murphy and Topel [1987a, 1987b] recognize these problems, and attempt to attenuate their impact in a variety of ways 
coefficients ${ }^{2}$ According to the unobserved ability explanation of industry and occupation wage effects, $A$ is correlated with the elements of $D$, in this case cross-section estimates of industry and occupation effects are biased

We use two intelligence test scores as error-prone measures of $A$ IQ and Knowledge of the World of Work (KWW) ${ }^{3}$ We assume that the IQ test score is related to ability through the equation,

$$
\mathrm{IQ}=A+\epsilon_{I},
$$

where $\epsilon_{I}$ is a measurement error uncorrelated with $A$ and with $\epsilon$ in (1) ${ }^{4}$ Equations (1) and (2) constitute the standard errors-invariables model One possible method for consistently estımating (1) and (2) is to instrument for IQ with the KWW score Suppose that KWW follows

$$
\mathrm{KWW}=\gamma_{K} A+\epsilon_{K}
$$

Assuming that $E\left(\epsilon_{I} \epsilon_{K}\right)=0$ and $E\left(\epsilon_{K} \epsilon\right)=0$, KWW 1s a valid instrument But this assumption will be violated if correlations in the test scores arise from test-takıng abilities (or other factors common across IQ and KWW scores) that are unrelated to $A$

An alternative way to identify the model is through an equation specifyng some of the determinants of $A$ Let $Z$ denote a vector of family background variables, such as parents' education, that may partly determine $A$ Consider the model consisting of (1), (2), and the auxiliary equation,

$$
A=Z \gamma_{Z}+\epsilon_{Z}
$$

Under the assumption that $E\left(\epsilon_{Z} \epsilon_{I}\right)=0$, the variables in $Z$ can serve as instrumental variables for IQ Alternatively, assuming that $E\left(\epsilon_{Z} \epsilon_{K}\right)=0$, the variables in $Z$ can serve as instruments when using KWW as a proxy for ability

Using family background variables to identify the model requires that these variables can be excluded from the wage

2 In the empirical work that follows, an early and later wage equation for each individual are estimated We use two wage observations so that we can check the consistency of our results across different points in the career path, and so that we can compute first-difference estimates for comparison with previous research

3 Detalls on these tests are given in Section III

4 Equation (2) may appear to suggest that the variance of IQ is necessarily higher than the variance of unobserved ability But the variance of the unobservable is identified by normalizing the coefficient of $A$ in equation (2) to equal one For example, true ability could have a much larger variance than $I Q$, but have a small coefficient in equation (2) 
equation There is an extensive debate in the returns-to-schooling literature on the effects of family background on earnings ${ }^{5}$ While there is a broad consensus that family background has important effects on schooling and ability, the direct effects of family background on earnings have generated more controversy Early research using simple recursive models for ablity, schooling, and earnings found that with the exception of parental income, family background variables such as parents' education, occupation, or number of siblings affect earnings only indirectly through ability and schooling [Duncan, 1968, Bowles and Nelson, 1974, Sewell and Hauser, 1975] But substantial measurement error in ability measures implies that alternative identifyng information is needed to test the exclusion of family background variables from wage equations ${ }^{6}$ One route is to choose some of the avalable background variables as valıd instruments a priori, and then to test the validity of the others But it is difficult to justify such distinctions among the avallable family background variables Alternatively, data on siblings (e g, Chamberlain and Grilıches [1977]) or twins (e g, Taubman [1976]) can provide identifyıng information, for example, a sibling's test score may be a valıd instrumental variable for the respondent's own test score Grilıches' [1979] research and review of this literature finds that family background variables appear to affect earnings primarily through their effect on schooling and ability, "[t]he market does not appear to pay for them drrectly" [p S59] Thus, observable family background variables are apparently valid instruments for ability measures in wage equations ${ }^{7}$

Of course, the techniques discussed in this section depend on the indicators of ability If test scores are actually unrelated to ability, or (more plausibly) if the unobserved ability that is rewarded in the labor market differs from the ability leading to higher test scores, then our methods cannot adequately test the unobserved ability hypothesis

\section{DATA}

Our principal source of data is the Young Men's Cohort of the National Longitudinal Survey (NLS) This cohort was first sur-

5 A thorough review is provided in Leibowitz [1977]

6 Griliches [1977] provides a review of this Literature

7 As a check on the robustness of our results, we also calculate the IV estimates that follow using one test score as an instrument for the other 
veyed in 1966 at ages 14-24, with 5,225 respondents, and resurveyed at one- or two-year intervals thereafter The sample we use is restricted to nonblack males The data set contans scores from two intelligence tests IQ and KWW The IQ scores were collected as part of a survey of the respondents' schools conducted in $1968^{8}$ Because respondents had to grant permission for schools to release IQ scores, and because school records were sometımes incomplete, IQ data are missing for about one third of the sample ${ }^{9}$ The KWW test examines respondents' knowledge about the labor market, covering the duties, educational attainment, and relative earnings of ten occupations ${ }^{10}$ While seemingly much different from an IQ test, Griliches [1976] found that least squares results for wage equations using IQ or KWW were quite similar

We study earnings and their determinants at two points first, the earliest year in which wages and other needed variables are avallable (though no later than 1973), and second, in 1980 The requirement that wages be observed at both points, the exclusion of individuals with missing IQ data, the restriction to nonblacks, and other data avalability requirements reduce the final sample size to $815^{11}$

There are three primary potential sources of selection bias in this subset of the original sample First, if IQ scores are missing nonrandomly (with respect to the wage equation error), then wage equation estimates based on the subsample for which IQ scores are avallable may be biased Second, an important determinant of whether an observation was avalable (particularly for the early years) was whether the individual had left school and gone to work, a decision likely to be related to labor market opportunities Grilıches, Hall, and Hausman [1978] address the influence of these potential sources of bias in wage equations estimated for the Young Men's Cohort of the NLS They cannot reject the hypothesis that the IQ data are randomly missing with respect to the error term in an equation for IQ ${ }^{12}$ Further, schooling and IQ coefficients in a

8 A wide variety of IQ tests are used in different states, these were combined on a consistent scale by the Center for Human Resources Research [1990], which administers the NLS

9 The KWW tests, in contrast, were administered as part of the initial survey, and hence are missing very infrequently

10 Further detals are given in Grilıches [1976]

11 An important source of missing data is incomplete records in the job histories that were used to construct a measure of actual experience

12 The test is based on a comparison of the sum of the likelihoods for an equation for IQ, estimated with OLS, and a probit for whether or not IQ data were avalable, to the likelihood for the joint model that accounts for selectivity 
wage equation are nearly identical whether they use the subsample for which IQ is avalable, or a sample in which missing IQ is filled in, takıng account of potential selectivity bias in the equation used to predict IQ ${ }^{13}$ As a further check on this source of blas, we examine the robustness of our findings in the larger sample for which KWW is avalable, but IQ is missing The results of Griliches, Hall, and Hausman also suggest that selectıvity into the working sample imparts a downward bias to schooling coefficients, and an upward bias to coefficients on IQ Ignoring this selection problem should then lead us to overstate the impact of unobserved ability on wages Together, the results from this earlier research suggest that these two sources of bias should not lead to spurious rejections of the unobserved ability explanation of interindustry and interoccupation wage differentials Finally, because we use information from 1980 , attrition bias may be significant ${ }^{14}$ Because of this, we examine the robustness of our results in a sample using data from only the early years of the survey

\section{EMPIRICAL Results}

Table I reports raw differences in log wages and test scores by industry and occupation, for both the early and 1980 observations These are computed from regressions of the dependent variables on a set of industry and occupation dummy variables A simple summary measure of the importance of industry and occupation coefficients is their standard deviation Unwelghted standard deviations are reported below the industry and occupation coefficient estimates ${ }^{15}$ There is substantial variation in the wages and test scores by both industry and occupation ( $F$-tests for equality of the means are rejected in all cases)

The bottom panel of the table reports correlations (as well as rank-order correlations) between these raw log wage and test score

13 While results are not reported for other wage equation coefficients (such as industry or occupation dummy variables, which were not included in their specifications), the high correlation between schooling and IQ makes it likely that any bias present would show up in the schooling coefficient

14 The NLS Young Men's Cohort had about 35 percent attrition by 1980 [Center for Human Resource Research, 1990]

15 The unweighted standard deviation measures the average "effect" of the industry or occupation coefficients for a randomly chosen industry or occupation, while the weighted (by employment) standard deviation would measure the effect for a randomly chosen individual The conclusions reached were not affected by using weighted standard deviations, or by correcting the standard deviations for sampling error 
differentials by industry and occupation The correlations of these differentials are often relatively high, six of the eight correlations are greater than 070 These estımates imply that, on average, there is a fairly high degree of correlation between average test scores and average (log) wages, both across industries (within occupations), and across occupations (within industries) ${ }^{16}$

As a prelıminary to estımatıng wage equations controlling for unobserved ability, in Table II we examine the extent to which differences in log wages and test scores by industry and occupation persist once the usual human capital controls are added The top panel of Table II reveals that interındustry wage differentials are scarcely diminished, whlle interoccupation wage differentials, as measured by their standard deviation, fall by close to one third ${ }^{17}$ Similarly, the addition of human capital controls does more to reduce the standard deviation of test score differentials across occupations than across industries

The bottom panel of Table II reports the correlations between these remaining industry and occupation differentials Just over half of these correlations are lower than the raw correlations in Table I, but the correlations remain quite large Thus, looking at average differences across industries or occupations, the unobserved ability explanation appears to recelve strong support However, these results are only suggestive regarding the determ1nants of wages at the individual level, where we ask whether the test scores (corrected for measurement error) are sufficiently strongly correlated with individuals' wages to reduce the magnitude of industry or occupation effects in individual-level wage regressions

Table III presents OLS and instrumental variables estimates of the early and late wage equations The OLS specifications include the test scores and the family background variables (later used as instruments) as independent variables in the wage equations, rather than relying on exclusion restrictions to correct the

16 In previous versions of this paper we reported correlations between wage differentials and test score differentials by industry only or by occupation only, rather than controlling for industry and occupation simultaneously Correlations calculated this way are considerably smaller, the difference reflects the concentration of workers in occupations with lower average wages (or test scores) in industries with lower average wages (or test scores)

17 The industry and occupation coefficients for the wage equations in Table II are similar to estimates from other data sets, both in terms of the magnitude and variation of industry and occupation differences, and in the ranking of industries and occupations as high- or low-wage 
TABLE I

RAW LOG WAGE, IQ, AND KWW DIFFERENCES By INDUSTRY AND OCCUPATION ${ }^{a}$

\begin{tabular}{|c|c|c|c|c|c|c|}
\hline & \multicolumn{3}{|c|}{ Early } & \multicolumn{3}{|c|}{1980} \\
\hline & $\begin{array}{c}\text { Log } \\
\text { wage }\end{array}$ & IQ & KWW & $\begin{array}{l}\text { Log } \\
\text { wage }\end{array}$ & IQ & KWW \\
\hline \multicolumn{7}{|l|}{ Industries } \\
\hline Minıng & 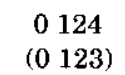 & $\begin{array}{r}-11138 \\
(4569)\end{array}$ & $\begin{array}{r}-0873 \\
(2502)\end{array}$ & $\begin{array}{cc}0 & 145 \\
\left(\begin{array}{lll}0 & 116\end{array}\right)\end{array}$ & $\begin{array}{c}0624 \\
(3901)\end{array}$ & 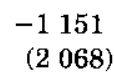 \\
\hline Construction & $\left.\begin{array}{rl}-0 & 083 \\
(0 & 072\end{array}\right)$ & $\begin{array}{r}-7654 \\
(2703)\end{array}$ & $\begin{array}{r}-3466 \\
(1480)\end{array}$ & $\left.\begin{array}{rl}-0 & 022 \\
(0 & 069\end{array}\right)$ & $\begin{array}{c}2850 \\
(2335)\end{array}$ & 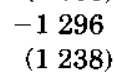 \\
\hline $\begin{array}{l}\text { Manufacturing-- } \\
\text { durables }\end{array}$ & $\begin{array}{rl}-0 & 019 \\
(0 & 063)\end{array}$ & $\begin{array}{r}-2493 \\
(2358)\end{array}$ & 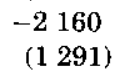 & 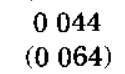 & $\begin{array}{c}1916 \\
(2154)\end{array}$ & $\begin{array}{r}-0967 \\
(1142)\end{array}$ \\
\hline $\begin{array}{c}\text { Manufacturing- } \\
\text { nondurables }\end{array}$ & $\begin{array}{rl}-0 & 101 \\
\left(\begin{array}{lll}0 & 064\end{array}\right)\end{array}$ & $\begin{array}{r}-4578 \\
(2372)\end{array}$ & $\begin{array}{r}-2528 \\
(1299)\end{array}$ & $\begin{array}{c}-0003 \\
(0066)\end{array}$ & $\begin{array}{c}1597 \\
(2216)\end{array}$ & $\begin{array}{r}-0238 \\
\left(\begin{array}{lll}1 & 175\end{array}\right)\end{array}$ \\
\hline $\begin{array}{l}\text { Transportation, } \\
\text { communication, } \\
\text { and public utlities }\end{array}$ & $\left.\begin{array}{rl}-0 & 029 \\
(0 & 070\end{array}\right)$ & $\begin{array}{r}-3130 \\
(2626)\end{array}$ & $\left.\begin{array}{rl}-1 & 363 \\
(1 & 438\end{array}\right)$ & $\begin{array}{cc}0 & 031 \\
(0 & 069)\end{array}$ & $\begin{array}{c}1968 \\
(2337)\end{array}$ & $\left.\begin{array}{rl}-1 & 222 \\
(1 & 239\end{array}\right)$ \\
\hline Trade & 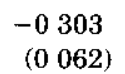 & $\begin{array}{r}-4774 \\
(2330)\end{array}$ & $\begin{array}{r}-3509 \\
(1276)\end{array}$ & $\begin{array}{c}-0200 \\
(0067)\end{array}$ & $\begin{array}{r}-1296 \\
(2269)\end{array}$ & 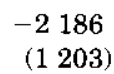 \\
\hline $\begin{array}{l}\text { Finance } \\
\text { and re }\end{array}$ & $\begin{array}{cc}-0 & 075 \\
(0 & 083)\end{array}$ & $\begin{array}{c}-0651 \\
(3077)\end{array}$ & $\begin{array}{r}-0751 \\
(1685)\end{array}$ & 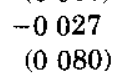 & $\begin{array}{c}2160 \\
(2692)\end{array}$ & $\begin{array}{r}-1250 \\
(1427)\end{array}$ \\
\hline $\begin{array}{l}\text { Business and } \\
\text { repair services }\end{array}$ & $\begin{array}{c}-0248 \\
(0088)\end{array}$ & $\begin{array}{rl}-4 & 103 \\
(3 & 267)\end{array}$ & $\begin{array}{r}-2387 \\
(1789)\end{array}$ & $\begin{array}{cc}0 & 085 \\
(0 & 097)\end{array}$ & $\begin{array}{c}0987 \\
(3249)\end{array}$ & $\begin{array}{r}-2073 \\
(1722)\end{array}$ \\
\hline Personal services & $\begin{array}{r}-0 \quad 323 \\
(0147)\end{array}$ & $\begin{array}{r}-7664 \\
(5484)\end{array}$ & $\begin{array}{r}-3437 \\
(3003)\end{array}$ & 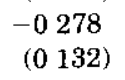 & $\begin{array}{r}-3503 \\
(4439)\end{array}$ & $\begin{array}{r}-6786 \\
(2353)\end{array}$ \\
\hline $\begin{array}{l}\text { Professional and } \\
\text { entertainment } \\
\text { services }\end{array}$ & $\begin{array}{r}-0229 \\
(0065)\end{array}$ & $\begin{array}{r}-4504 \\
(2434)\end{array}$ & $\begin{array}{r}-2380 \\
(1333)\end{array}$ & 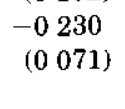 & 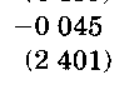 & $\left.\begin{array}{rl}-1 & 465 \\
(1 & 273\end{array}\right)$ \\
\hline $\begin{array}{l}\text { Standard deviation } \\
\text { of coefficients }\end{array}$ & 0141 & 3242 & 1194 & 0136 & 1828 & 1810 \\
\hline \multicolumn{7}{|l|}{ Occupations } \\
\hline $\begin{array}{l}\text { Professional, tech- } \\
\text { nical, and kindred } \\
\text { workers }\end{array}$ & $\begin{array}{c}0 \quad 343 \\
\left(\begin{array}{lll}0 & 057\end{array}\right)\end{array}$ & $\begin{array}{l}11919 \\
(2088)\end{array}$ & $\begin{array}{c}7375 \\
(1 \quad 144)\end{array}$ & $\begin{array}{c}0376 \\
\left(\begin{array}{lll}0 & 092\end{array}\right)\end{array}$ & $\begin{array}{l}14924 \\
(3081)\end{array}$ & $\begin{array}{r}4199 \\
(11633)\end{array}$ \\
\hline $\begin{array}{l}\text { Managers, officials, } \\
\text { and proprietors }\end{array}$ & $\begin{array}{c}0263 \\
(0264)\end{array}$ & $\begin{array}{c}7528 \\
(2349)\end{array}$ & $\begin{array}{c}6708 \\
(1286)\end{array}$ & $\begin{array}{c}0499 \\
\left(\begin{array}{lll}0 & 091)\end{array}\right)\end{array}$ & $\begin{array}{l}11814 \\
(3050)\end{array}$ & $\begin{array}{r}5824 \\
(1617)\end{array}$ \\
\hline $\begin{array}{l}\text { Clerical and kindred } \\
\text { workers }\end{array}$ & $\left.\begin{array}{cc}0 & 044 \\
(0 & 060\end{array}\right)$ & $\begin{array}{c}1087 \\
(2203)\end{array}$ & $\begin{array}{c}3886 \\
(1207)\end{array}$ & $\begin{array}{ll}0 & 128 \\
(0 & 097)\end{array}$ & $\begin{array}{c}9584 \\
(3278)\end{array}$ & $\begin{array}{c}0928 \\
(1738)\end{array}$ \\
\hline Sales workers & $\begin{array}{c}0218 \\
\left(\begin{array}{lll}0 & 069\end{array}\right)\end{array}$ & $\begin{array}{c}3626 \\
(2551)\end{array}$ & $\begin{array}{c}6752 \\
(1397)\end{array}$ & $\begin{array}{c}0325 \\
(0 \quad 103)\end{array}$ & $\begin{array}{l}10576 \\
(3467)\end{array}$ & $\begin{array}{c}4045 \\
(1 \quad 838)\end{array}$ \\
\hline $\begin{array}{l}\text { Craftsmen, foremen, } \\
\text { and kındred workers }\end{array}$ & $\left.\begin{array}{cc}0 & 106 \\
(0 & 053)\end{array}\right)$ & $\begin{array}{r}-0356 \\
(1 \quad 941)\end{array}$ & $\begin{array}{c}2890 \\
(1063)\end{array}$ & $\begin{array}{c}0218 \\
(0088)\end{array}$ & $\begin{array}{c}2939 \\
(2954)\end{array}$ & $\begin{array}{rl}-0 & 184 \\
(1 & 566)\end{array}$ \\
\hline Operatives and & $\begin{array}{c}0028 \\
(0052)\end{array}$ & $\begin{array}{l}-1305 \\
(1922)\end{array}$ & $\begin{array}{c}2261 \\
(1052)\end{array}$ & $\begin{array}{ccc}0 & 060 \\
(0 & 090)\end{array}$ & $\begin{array}{c}1862 \\
(3040)\end{array}$ & $\begin{array}{r}-1806 \\
(1611)\end{array}$ \\
\hline Service workers & 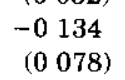 & $\begin{array}{c}-3679 \\
(2904)\end{array}$ & $\begin{array}{c}0038 \\
(1590)\end{array}$ & $\left.\begin{array}{ccc}0 & 074 \\
(0 & 112\end{array}\right)$ & $\begin{array}{l}8946 \\
(3782)\end{array}$ & $\begin{array}{c}-0663 \\
(2005)\end{array}$ \\
\hline $\begin{array}{l}\text { Standard deviation } \\
\text { of coefficients }\end{array}$ & 0157 & 5129 & 2969 & 0176 & 5324 & 2764 \\
\hline
\end{tabular}


TABLE I

(CONTINUED)

\begin{tabular}{|c|c|c|c|c|c|c|}
\hline \multicolumn{7}{|c|}{ Correlations of Raw Log Wage and Test Score Differentials } \\
\hline & & & Industry & & & \\
\hline & \multicolumn{2}{|c|}{ Early } & & & \multicolumn{2}{|c|}{1980} \\
\hline & IQ & KWW & & & IQ & KWW \\
\hline \multirow[t]{3}{*}{ Log wage } & $\begin{array}{cc}-0 & 01 \\
{\left[\begin{array}{ll}0 & 35\end{array}\right]}\end{array}$ & 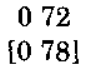 & & Log wage & 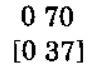 & $\begin{array}{c}062 \\
{\left[\begin{array}{ll}0 & 55\end{array}\right]}\end{array}$ \\
\hline & & & Occupation & & & \\
\hline & IQ & KWW & & & IQ & KWW \\
\hline Log wage & $\begin{array}{c}094 \\
{\left[\begin{array}{ll}0 & 88\end{array}\right]}\end{array}$ & $\begin{array}{c}094 \\
{\left[\begin{array}{ll}0 & 93\end{array}\right]}\end{array}$ & & Log wage & $\begin{array}{c}074 \\
{\left[\begin{array}{ll}0 & 90\end{array}\right]}\end{array}$ & $\begin{array}{c}092 \\
{\left[\begin{array}{l}0 \\
01\end{array}\right]}\end{array}$ \\
\hline
\end{tabular}

a Coefficient estimates from regressions of log wage IQ, and KWW on industry and occupation dummy varuables, with no other control variables except that in the early log wage regression dummy variables are included for the year from which the observation was drawn Public administration is the omitted industry Laborers is the omitted occupation There are 815 observations Standard errors of coeficient estimates are reported in parentheses

b Standard Pearson correlations are reported in the first row Spearman rank order correlations are reported in square brackets

test score coefficients for measurement error If there is substantial measurement error in the test scores, the reduction in the estimated standard deviation of the industry and occupation coefficients-when we include the test scores-will be understated in these specifications ( $\mathrm{On}$ the other hand, including the family background variables may partly offset the effect of the measurement error ) Compared with the estimates from Table II, the standard deviation of the industry effects rises shightly for the early equation (to 0142 ), and falls slightly for the 1980 equation (to 0 126) The standard deviation of the occupation effects declines by about $001 \mathrm{in}$ both cases Thus, these results provide little or no support for the unobserved abllity explanation of industry or occupation wage differentials

For the IV estımates, the model was estımated as a system that includes the two wage equations and a test score equation Two sets of estimates are reported, one using IQ as the indicator of ablity, and another using KWW The set of family background variables used as instruments is the same in each specification In all of the specifications, ability enters significantly, with coefficients roughly five to ten times the magnitude obtained in the OLS estimates without the measurement error correction The standard deviations of the industry coefficients fall by about 10 to 15 percent, relative to the OLS estimates from Table II, while the 
TABLE II

LOG WAGE, IQ, AND KWW DIFFERENCES BY INDUSTRY AND OCCUPATION, INCLUDING WAGE REGRESSION CONTROLS ${ }^{a}$

\begin{tabular}{|c|c|c|c|c|c|c|}
\hline & \multicolumn{3}{|c|}{ Early } & \multicolumn{3}{|c|}{1980} \\
\hline & $\begin{array}{c}\text { Log } \\
\text { wage }\end{array}$ & IQ & KWW & $\begin{array}{c}\text { Log } \\
\text { wage }\end{array}$ & $\mathrm{IQ}$ & KWW \\
\hline \multicolumn{7}{|l|}{ Industries } \\
\hline Minıng & 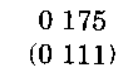 & $\begin{array}{r}-5801 \\
(4261)\end{array}$ & $\begin{array}{c}-0769 \\
(2376)\end{array}$ & $\begin{array}{c}0168 \\
(0106)\end{array}$ & $\begin{array}{l}2144 \\
(3529)\end{array}$ & $\begin{array}{rl}-1 & 130 \\
(1 & 947)\end{array}$ \\
\hline Construction & $\begin{array}{rl}-0 & 029 \\
(0 & 065)\end{array}$ & $\begin{array}{r}-4537 \\
(2504)\end{array}$ & $\begin{array}{rl}-2 & 541 \\
(1 & 397)\end{array}$ & 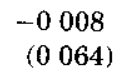 & $\begin{array}{l}2875 \\
(2117)\end{array}$ & $\begin{array}{r}-0694 \\
(1 \quad 168)\end{array}$ \\
\hline $\begin{array}{l}\text { Manufacturing- } \\
\text { durables }\end{array}$ & $\begin{array}{rl}-0 & 013 \\
(0 & 057)\end{array}$ & 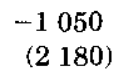 & $\begin{array}{r}-1747 \\
(1216)\end{array}$ & 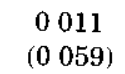 & $\begin{array}{l}1693 \\
(1955)\end{array}$ & $\left.\begin{array}{rl}-1 & 165 \\
(1 & 079\end{array}\right)$ \\
\hline $\begin{array}{c}\text { Manufacturing- } \\
\text { nondurables }\end{array}$ & $\begin{array}{rl}-0 & 062 \\
\left(\begin{array}{ll}0 & 057\end{array}\right)\end{array}$ & 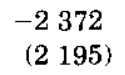 & $\begin{array}{r}-1578 \\
(1224)\end{array}$ & 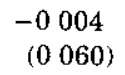 & $\begin{array}{l}1277 \\
(2006)\end{array}$ & 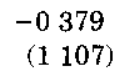 \\
\hline $\begin{array}{l}\text { Transportation, } \\
\text { communication, } \\
\text { and public utilities }\end{array}$ & 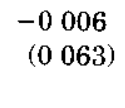 & $\begin{array}{r}-1938 \\
(2422)\end{array}$ & 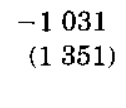 & $\begin{array}{c}0037 \\
(0064)\end{array}$ & $\begin{array}{l}2940 \\
(2116)\end{array}$ & 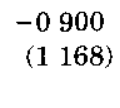 \\
\hline Trade & $\begin{array}{rl}-0 & 277 \\
(0 & 056)\end{array}$ & $\begin{array}{r}-2965 \\
(2155)\end{array}$ & $\begin{array}{r}-2870 \\
(1202)\end{array}$ & $\begin{array}{rl}-0 & 201 \\
(0 & 062)\end{array}$ & $\begin{array}{rl}-1 & 261 \\
(2053)\end{array}$ & $\left.\begin{array}{rl}-1 & 930 \\
(1 & 133\end{array}\right)$ \\
\hline $\begin{array}{l}\text { Finance, ins } \\
\text { and real es }\end{array}$ & 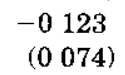 & $\begin{array}{r}-2533 \\
(2841)\end{array}$ & $\begin{array}{r}-1449 \\
(1584)\end{array}$ & $\begin{array}{r}-0 \\
(0\end{array}$ & $\begin{array}{r}1 \\
(2\end{array}$ & $\begin{array}{rl}-1 & 545 \\
(1 & 345)\end{array}$ \\
\hline $\begin{array}{c}\text { Business } \\
\text { repair }\end{array}$ & 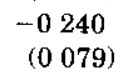 & $\begin{array}{r}-1244 \\
(3024)\end{array}$ & $\left.\begin{array}{rl}-2 & 052 \\
(1 & 686\end{array}\right)$ & 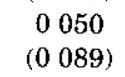 & $\begin{array}{c}0370 \\
(2945)\end{array}$ & $\begin{array}{rl}-1 & 931 \\
(1 & 625)\end{array}$ \\
\hline Personal services & $\begin{array}{r}-0249 \\
(0132)\end{array}$ & $\begin{array}{r}-6783 \\
(5070)\end{array}$ & $\begin{array}{rl}-2 & 491 \\
(2 & 828)\end{array}$ & $\begin{array}{r}-0211 \\
(0121)\end{array}$ & 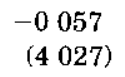 & $\begin{array}{r}-5484 \\
(2222)\end{array}$ \\
\hline $\begin{array}{l}\text { Professional and } \\
\text { entertainment } \\
\text { services }\end{array}$ & 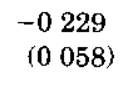 & $\begin{array}{r}-6024 \\
(2247)\end{array}$ & $\begin{array}{r}-2649 \\
(1253)\end{array}$ & $\begin{array}{l}-0268 \\
(0066)\end{array}$ & $\begin{array}{r}-3139 \\
(2183)\end{array}$ & $\begin{array}{r}-2017 \\
(1204)\end{array}$ \\
\hline $\begin{array}{l}\text { Standard deviation } \\
\text { of coefficients }\end{array}$ & 0141 & 2255 & 0894 & 0131 & 1833 & 1457 \\
\hline \multicolumn{7}{|l|}{ Occupations } \\
\hline $\begin{array}{l}\text { Professional, tech- } \\
\text { nical, and kındred } \\
\text { workers }\end{array}$ & $\begin{array}{cc}0 & 187 \\
(0 & 054)\end{array}$ & $\begin{array}{c}5041 \\
(2053)\end{array}$ & $\begin{array}{l}3953 \\
(1145)\end{array}$ & $\begin{array}{c}0239 \\
\left(\begin{array}{lll}0 & 085\end{array}\right)\end{array}$ & $\begin{array}{l}9169 \\
(2831)\end{array}$ & $\begin{array}{l}1978 \\
(1562)\end{array}$ \\
\hline $\begin{array}{l}\text { Managers, officials, } \\
\text { and proprietors }\end{array}$ & $\begin{array}{c}0 \\
\left.\begin{array}{lll}0 & 125 \\
0 & 058\end{array}\right)\end{array}$ & $\begin{array}{c}3676 \\
(2239)\end{array}$ & $\begin{array}{c}3557 \\
(1249)\end{array}$ & $\begin{array}{c}0382 \\
\left(\begin{array}{lll}0 & 084\end{array}\right)\end{array}$ & $\begin{array}{c}7909 \\
(2784)\end{array}$ & $\begin{array}{c}3761 \\
(1536)\end{array}$ \\
\hline $\begin{array}{l}\text { Clerical and kındred } \\
\text { workers }\end{array}$ & 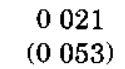 & 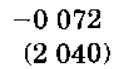 & $\begin{array}{c}3081 \\
(1138)\end{array}$ & $\left.\begin{array}{cc}0 & 105 \\
(0 & 090\end{array}\right)$ & $\begin{array}{c}7370 \\
(2977)\end{array}$ & $\begin{array}{c}0361 \\
(1643)\end{array}$ \\
\hline Sales workers & $\begin{array}{c}0137 \\
(0063)\end{array}$ & $\begin{array}{c}0268 \\
(2386)\end{array}$ & $\begin{array}{rl}4 & 706 \\
(1331)\end{array}$ & $\begin{array}{c}0242 \\
\left(\begin{array}{lll}0 & 095\end{array}\right)\end{array}$ & $\begin{array}{c}7459 \\
(3156)\end{array}$ & $\begin{array}{c}2489 \\
(1742)\end{array}$ \\
\hline $\begin{array}{l}\text { Craftsmen, foremen, } \\
\text { and kındred workers }\end{array}$ & $\left.\begin{array}{cc}0 & 050 \\
(0 & 048\end{array}\right)$ & $\begin{array}{c}0915 \\
(1823)\end{array}$ & $\begin{array}{c}1734 \\
(1017)\end{array}$ & $\begin{array}{c}0259 \\
\left(\begin{array}{lll}0 & 081\end{array}\right)\end{array}$ & $\begin{array}{c}5573 \\
(2679)\end{array}$ & $\left.\begin{array}{rl}-0 & 053 \\
(1 & 479\end{array}\right)$ \\
\hline $\begin{array}{l}\text { Operatives and } \\
\text { kındred workers }\end{array}$ & 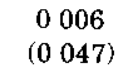 & $\begin{array}{c}0054 \\
(1782)\end{array}$ & $\begin{array}{c}1889 \\
(0994)\end{array}$ & $\begin{array}{cc}0 & 132 \\
\left(\begin{array}{lll}0 & 083\end{array}\right)\end{array}$ & $\begin{array}{c}4512 \\
(2761)\end{array}$ & $\begin{array}{rl}-1 & 092 \\
(1 & 524)\end{array}$ \\
\hline Service workers & $\left.\begin{array}{rl}-0 & 136 \\
(0 & 070\end{array}\right)$ & $\begin{array}{r}-1650 \\
(2687)\end{array}$ & $\left.\begin{array}{rl}-0 & 059 \\
(1 & 498\end{array}\right)$ & $\left.\begin{array}{ccc}0 & 069 \\
(0 & 103\end{array}\right)$ & $\begin{array}{c}8843 \\
(3424)\end{array}$ & $\begin{array}{rl}-0 & 493 \\
(1 & 890)\end{array}$ \\
\hline $\begin{array}{l}\text { Standard deviation } \\
\text { of coefficients }\end{array}$ & 0101 & 2207 & 1774 & 0124 & 3004 & 1681 \\
\hline
\end{tabular}


TABLE II

(CONTINUED)

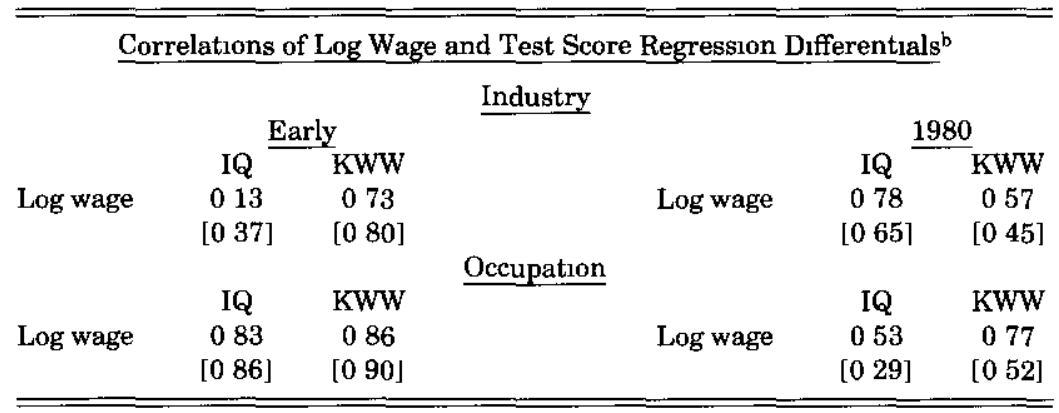

a Coefficient estimates from regressions of log wage IQ, and KWW on industry and occupation dummy variables Other control variables included in regressions are years of schooling actual labor market experience (and sts square in the 1980 regressions), dummy variables for married, spouse present and residence in the South and in an SMSA and in the early log wage regression dummy variables for the year from which the observation was drawn Public adminstration is the omitted industry Laborers is the omitted occupation There are 815 observations Standard errors of coefficient estımates are reported in parentheses

b Standard Pearson correlations are reported in the first row Spearman rank-order correlations are reported in square brackets

standard deviations of the occupation coefficients fall by about 15 to 30 percent ${ }^{18}$

These results were replicated with full information maximum likelihood estimates of the model, using both IQ and KWW as indicators of ability, employing the LISREL program [Joreskog and Sorbom, 1984], compared with the IV estımates, the maximum likelihood estimates indicated a smaller decline in the standard deviations of the industry and occupation effects, relative to the OLS estımates in Table II We also verified that the results were not sensitive to alternative variable definitions, sample definitions, or model specifications These robustness checks included using two-digit instead of one-digt industries, expanding the sample to include observations missing IQ data, using KWW as the only ability indicator, excluding indıviduals in the mining industry (who may receive a significant compensating differential), estımating a model with two ability factors, and restricting the analysis to data drawn only from the years 1966-1973, to minimize attrition bias 19

Consequently, we conclude that individual-level results such as those in Table III do not support the unobserved ability

18 When we used KWW as the instrument for $1 \mathrm{~K}$, or $\mathrm{IQ}$ as the instrument for KWW, the estimated reductions in the standard deviations of the industry and occupation effects were within the same ranges

19 Most of these results are provided in an earlier version of the paper All results are avalable from the authors upon request 
TABLE III

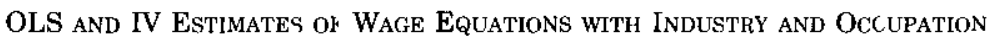
DUMMY VARIABLE $\varsigma^{\mathrm{a}}$

\begin{tabular}{|c|c|c|c|c|c|c|}
\hline & $\begin{array}{l}\text { Earlyb }^{b} \\
\text { OLS }\end{array}$ & $\begin{array}{l}\text { Early } \\
\text { IQ as } \\
\text { proxy } \\
\text { IV }\end{array}$ & $\begin{array}{c}\text { Early } \\
\text { KWW as } \\
\text { proxyc }^{\mathrm{c}} \\
\text { IV }\end{array}$ & $\begin{array}{c}1980^{b} \\
\text { OLS }\end{array}$ & $\begin{array}{l}1980 \\
\text { IQ as } \\
\text { proxy }^{\mathrm{c}} \\
\text { IV }\end{array}$ & $\begin{array}{c}1980 \\
\text { KWW as } \\
\text { proxy }^{c} \\
\text { IV }\end{array}$ \\
\hline \multicolumn{7}{|l|}{ Industries } \\
\hline$\overline{\text { Mining }}$ & $\begin{array}{c}0196 \\
\left(\begin{array}{ll}0 & 110\end{array}\right)\end{array}$ & 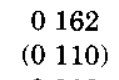 & $\begin{array}{c}0151 \\
\left(\begin{array}{lll}0 & 107\end{array}\right)\end{array}$ & 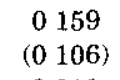 & $\begin{array}{c}0132 \\
(0104)\end{array}$ & 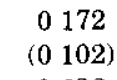 \\
\hline Construction & $\begin{array}{r}-0015 \\
(0064)\end{array}$ & $\begin{array}{l}-0018 \\
(0066)\end{array}$ & 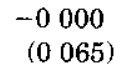 & 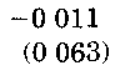 & 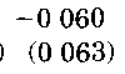 & 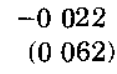 \\
\hline $\begin{array}{l}\text { Manufacturing- } \\
\text { durables }\end{array}$ & 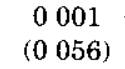 & $\begin{array}{rl}-0 & 020 \\
(0 & 055)\end{array}$ & 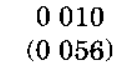 & $\begin{array}{c}0011 \\
(0059)\end{array}$ & $\begin{array}{c}-0030 \\
(0058)\end{array}$ & $\begin{array}{l}0006 \\
(0057)\end{array}$ \\
\hline $\begin{array}{c}\text { Manufacturing- } \\
\text { nondurables }\end{array}$ & $\left.\begin{array}{rl}-0 & 043 \\
(0 & 056\end{array}\right)$ & $\begin{array}{rl}-0 & 053 \\
(0 & 056)\end{array}$ & 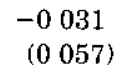 & 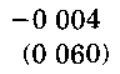 & 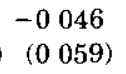 & $\begin{array}{rl}-0 & 025 \\
(0 & 058)\end{array}$ \\
\hline $\begin{array}{l}\text { Transportation, } \\
\text { communication, } \\
\text { and public utılities }\end{array}$ & $\left.\begin{array}{ccc}0 & 0 & 02 \\
(0 & 062\end{array}\right)$ & $\begin{array}{c}-0008 \\
(0062)\end{array}$ & 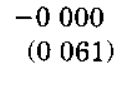 & 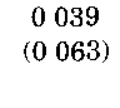 & $\begin{array}{c}-0001 \\
(0063)\end{array}$ & $\begin{array}{rl}0 & 038 \\
(0 & 062)\end{array}$ \\
\hline Trade & $\begin{array}{r}-0260 \\
(0055)\end{array}$ & $\begin{array}{r}-0246 \\
(0056)\end{array}$ & 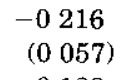 & $\begin{array}{cc}-0 & 193 \\
(0 & 062)\end{array}$ & $\begin{array}{c}-0204 \\
(0060)\end{array}$ & $\left.\begin{array}{rl}-0 & 184 \\
(0 & 060\end{array}\right)$ \\
\hline $\begin{array}{l}\text { Finance, msurance, } \\
\text { and real estate }\end{array}$ & $\begin{array}{rl}-0 & 114 \\
(0 & 073)\end{array}$ & $\begin{array}{rl}-0 & 136 \\
(0 & 073)\end{array}$ & $\begin{array}{rl}-0 & 123 \\
\left(\begin{array}{lll}0 & 072\end{array}\right)\end{array}$ & 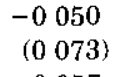 & $\begin{array}{cc}-0 & 105 \\
\left(\begin{array}{ll}0 & 072\end{array}\right)\end{array}$ & 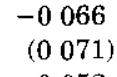 \\
\hline $\begin{array}{l}\text { Business and } \\
\text { repair services }\end{array}$ & $\begin{array}{r}-0226 \\
(0077)\end{array}$ & $\begin{array}{l}-0245 \\
(0076)\end{array}$ & $\begin{array}{rl}-0 & 219 \\
(0 & 077)\end{array}$ & $\begin{array}{c}0057 \\
(0088)\end{array}$ & $\begin{array}{c}0020 \\
(0086)\end{array}$ & $\begin{array}{rl}0 & 052 \\
(0 & 085)\end{array}$ \\
\hline Personal services & $\begin{array}{r}-0259 \\
(0131)\end{array}$ & $\begin{array}{r}-0216 \\
(0131)\end{array}$ & $\left.\begin{array}{rl}-0 & 236 \\
(0 & 127\end{array}\right)$ & $\begin{array}{r}-0204 \\
(0121)\end{array}$ & 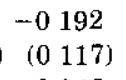 & $\left.\begin{array}{rl}-0 & 114 \\
(0 & 119\end{array}\right)$ \\
\hline $\begin{array}{l}\text { Professional and } \\
\text { entertainment } \\
\text { services }\end{array}$ & $\begin{array}{rl}-0 & 199 \\
(0 & 058)\end{array}$ & $\begin{array}{rl}-0 & 184 \\
(0 & 061)\end{array}$ & 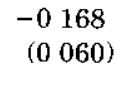 & $\begin{array}{rl}-0 & 256 \\
(0 & 066)\end{array}$ & $\begin{array}{c}-0236 \\
(0265)\end{array}$ & $\begin{array}{rl}-0 & 230 \\
(0 & 064)\end{array}$ \\
\hline $\begin{array}{l}\text { Standard deviation } \\
\text { of coefficients }\end{array}$ & 0142 & 0128 & 0124 & 0126 & 0111 & 0112 \\
\hline \multicolumn{7}{|l|}{ Occupations } \\
\hline $\begin{array}{l}\text { Professional, tech- } \\
\text { nical, and kundred } \\
\text { workers }\end{array}$ & $\left.\begin{array}{cc}0 & 155 \\
(0 & 054\end{array}\right)$ & $\begin{array}{c}0128 \\
(0055)\end{array}$ & $\begin{array}{c}0107 \\
\left(\begin{array}{lll}0 & 055\end{array}\right)\end{array}$ & $\begin{array}{c}0214 \\
(0085)\end{array}$ & 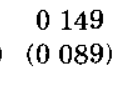 & $\begin{array}{c}0191 \\
(0082)\end{array}$ \\
\hline $\begin{array}{l}\text { Managers, officials, } \\
\text { and proprietors }\end{array}$ & 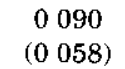 & $\begin{array}{cc}0 & 065 \\
(0 & 058)\end{array}$ & 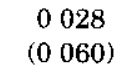 & 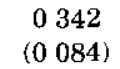 & $\begin{array}{c}0282 \\
(0086)\end{array}$ & $\begin{array}{c}0291 \\
(02082)\end{array}$ \\
\hline $\begin{array}{l}\text { Clerical and kındred } \\
\text { workers }\end{array}$ & $\left.\begin{array}{ccc}0 & 0 & 01 \\
(0 & 053\end{array}\right)$ & $\begin{array}{c}-0009 \\
(0052)\end{array}$ & $\begin{array}{rl}-0 & 046 \\
(0 & 053)\end{array}$ & $\left.\begin{array}{ll}0 & 097 \\
(0 & 089\end{array}\right)$ & $\begin{array}{c}0054 \\
(0091)\end{array}$ & $\left.\begin{array}{cc}0 & 105 \\
(0 & 086\end{array}\right)$ \\
\hline Sales workers & $\left.\begin{array}{cc}0 & 102 \\
(0 & 062\end{array}\right)$ & 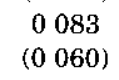 & 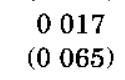 & $\begin{array}{l}0224 \\
(0095)\end{array}$ & $\begin{array}{ccc}0 & 176 \\
(0 & 096)\end{array}$ & 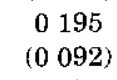 \\
\hline $\begin{array}{l}\text { Craftsmen, foremen, } \\
\text { and kindred workers }\end{array}$ & $\begin{array}{cc}0 & 041 \\
\left(\begin{array}{lll}0 & 047\end{array}\right)\end{array}$ & 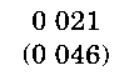 & 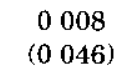 & $\begin{array}{c}0244 \\
(0208)\end{array}$ & $\begin{array}{c}0209 \\
(0281)\end{array}$ & $\begin{array}{l}0251 \\
(0077)\end{array}$ \\
\hline $\begin{array}{l}\text { Operatives and } \\
\text { kındred workers }\end{array}$ & $\begin{array}{rl}-0 & 006 \\
(0 & 046)\end{array}$ & 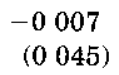 & $\left.\begin{array}{rl}-0 & 022 \\
(0 & 045\end{array}\right)$ & $\begin{array}{cc}0 & 134 \\
\left(\begin{array}{lll}0 & 083\end{array}\right)\end{array}$ & 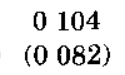 & $\begin{array}{c}0148 \\
(0080)\end{array}$ \\
\hline Service workers & $\left.\begin{array}{rl}-0 & 132 \\
(0 & 069\end{array}\right)$ & $\left.\begin{array}{rl}-0 & 138 \\
(0 & 068\end{array}\right)$ & $\left.\begin{array}{rl}-0 & 139 \\
(0 & 068\end{array}\right)$ & 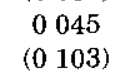 & 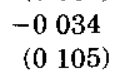 & $\left.\begin{array}{ll}0 & 040 \\
(0 & 099\end{array}\right)$ \\
\hline $\begin{array}{l}\text { Standard deviation } \\
\text { of coefficients }\end{array}$ & 0088 & 0080 & 0070 & 0114 & 0108 & 0100 \\
\hline
\end{tabular}


TABLE III

(CONTINUED)

\begin{tabular}{|c|c|c|c|c|c|c|}
\hline & $\begin{array}{c}\text { Earlyb }^{\mathbf{b}} \\
\text { OLS }\end{array}$ & $\begin{array}{c}\text { Early } \\
\text { IQ as } \\
\text { proxyc } \\
\text { IV }\end{array}$ & $\begin{array}{c}\text { Early } \\
\text { KWW as } \\
\text { proxyc }^{c} \\
\text { IV }\end{array}$ & $\begin{array}{l}1980^{\mathrm{b}} \\
\text { OLS }\end{array}$ & $\begin{array}{c}1980 \\
\text { IQ as } \\
\text { proxyc } \\
\text { IV }\end{array}$ & $\begin{array}{c}1980 \\
\text { KWW as } \\
\text { proxy }^{\mathrm{c}} \\
\text { IV }\end{array}$ \\
\hline \multicolumn{7}{|l|}{ Other controls } \\
\hline Ability (IQ) & 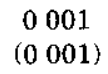 & 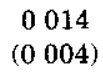 & & 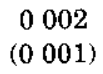 & 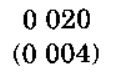 & \\
\hline Abllity (KWW) & 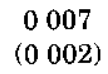 & & 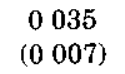 & 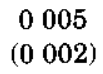 & & 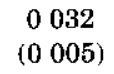 \\
\hline Schooling & 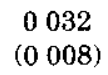 & 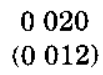 & 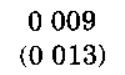 & 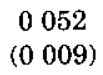 & 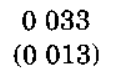 & 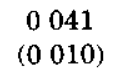 \\
\hline$R^{2}$ & 0540 & & & 0326 & & \\
\hline
\end{tabular}

a Public administration is the omitted industry Laborers 1s the ornited occupation There are 815 observations Control variables described in footnotes to Table JI are included in all specifications Standard errors of coefficient estimates are reported in parentheses

b Family background variables are also included in regressions The family background variables include number of siblings birth order, father's education mother's education and dummy variables for missing data for these varuables

c The family background variables listed in footnote 2 are excluded trom these specifications and used as instrumental variables

explanation of interindustry and (to a lesser extent) interoccupation wage differentials Can this conclusion be reconciled with the apparently contradictory industry- and occupation-level results in Tables I and II? One interpretation is that the test scores that we use are only partly correlated with other types of ability that are rewarded in labor markets, looking at average differentials by industry or occupation may do more to reduce the effects of measurement error than does instrumenting with family background measures However, this argument implies that results with a richer set of test scores would lead to larger reductions in industry and occupation effects in individual-level wage regressions To examine this question, we reestimated the equations in Table III using data from the National Longitudinal Survey Youth Cohort, which contains a richer set of test scores ${ }^{20}$ Estimates with these data yielded reductions in the standard deviations of industry and occupation effects similar to those reported in Table III This strengthens the conclusion that ability as measured by a variety of

20 The NLS Youth Cohort data set includes scores on the Armed Services Vocational Aptitude Battery, a set of tests of both academic and technical ability and knowledge These results are provided in an earlier version of the paper, available from the authors upon request 
test scores cannot explain interindustry and interoccupation wage differentials in standard cross-section wage regressions

Our finding that omitted ability does not appear to significantly bias estimated industry effects contrasts with the modified first-difference findıngs of Murphy and Topel [1987a, 1987b], who reject the "pure" Industry effects hypothesis in favor of the unobserved ability hypothesis ${ }^{21}$ But it is consistent with the measurement-error-corrected results in Krueger and Summers [1988], and with first-difference results for workers with exogenous separations (thus reducing bias from endogenous selection) in Gibbons and Katz [1992] The Murphy and Topel [1987b] results may differ for a number of reasons First, they use a weekly wage defined as annual earnings divided by annual weeks, on all jobs worked over the course of the year Because earnings on both the origin and destination job for industry or occupation changers are included in this measure, wage changes are likely to be understated ${ }^{22}$ In addition, although Murphy and Topel use the CPS, their sample may be unrepresentative, since they are able to use only those individuals who do not change residence when changing industry To obtain a rough check on this, we estimated a first-difference model similar to theirs, using our early and late observations ${ }^{23}$ The resulting estimates are more consistent with the unobserved ability explanation of interindustry and interoccupation wage differentials than the estimates in Table III, but not nearly so much as Murphy and Topel's estımates ${ }^{24}$ Furthermore, results with our data indicate that this modified estimator and the standard first-difference estımator yıeld simılar results ${ }^{25}$ Thus,

21 Murphy and Topel include cross-sectional OLS estımates of industry effects for each individual in a first-difference specification The coefficients of these variables can be used to test the pure industry effects hypothesis (for which the coefficient should equal one) against the unobserved ability hypothesis (for which the coefficient should equal zero) Their estimate of the industry coefficient is 027 They estimate a similar occupation effects coefficient of 008

22 This problem was pointed out by a referee

23 We computed the industry and occupation effects from the full sample, whereas Murphy and Topel [1987b] use only the nonmovers In our case, the number of nonmovers would be very small (233), and would likely be a highly select sample, since the interval between observations is so long

24 Our estimates of the industry and occupation coefficients (standard errors) are $0637(0126)$ and $0429(0145)$

25 Our first-difference results may differ from those of Murphy and Topel not only because of the way they construct the wage, but also because of the longer period of time over which changes are recorded in our data (on average more than ten years, compared with one year in the Murphy and Topel papers), suggesting that a higher proportion of reported changers are true changers In our sample 71 percent of the respondents change industry, compared with 4 percent in their sample As a result, measur ement-error bias is likely to be more severe in their sample [Freeman, 1984] Recognizing this, Murphy and Topel use an instrumental variables approach 
the divergence in results appears to be attributable to differences in the definition of the wage, the sample used, and the effects of measurement error

\section{CONCLUSION}

In this paper we test the unobserved ablity explanation of interindustry and interoccupation wage differentials by explicitly incorporating measures of unobserved ability into wage regressions The procedure we use may be an improvement over past attempts to account for unobserved ability using standard firstdifference estimators, since it is less likely to suffer from biases due to measurement error or selectivity The major limitation of our approach is that we cannot control for variation in ability that is not reflected in the test scores that we use as indicators of ability Our empirical results imply that interindustry and interoccupation wage differentials are, for the most part, not attributable to variation in unobserved labor quality or ablity Our estımates indicate that just over one tenth of the variation in interindustry wage differentials, and less than one fourth of the variation in interoccupation wage differentials, reflect differences in unobserved ability

UNIVERSITY OF SOUTH CAROLINA

UNiversity of PenNsylvania and National BuREaU of Economic RESEARCh

\section{REFERENCES}

Bowles, Samuel, and Valerie I Nelson, "The Inheritance of I Q and the Intergenerational Reproduction of Economic Inequality," Review of Economics and Statistics, LVI (1974), 39-51

Center for Human Resources Research, NLS Handbook (Columbus, OH The Ohio State University, 1990)

Chamberlain, Gary, "Education, Income, and Abılity Revisited," Journal of Econometrics, V (1977), 241-57

Chamberlain, Gary, and Zv1 Griliches, "More on Brothers," in Paul Taubman, ed, Kinometrics Determinants of Socioeconomic Success Within and Between Families (Amsterdam North-Holland Publishing Company, 1977)

Corcoran, Mary, Christopher Jencks, and Michael Olneck," The Effects of Family Background on Earnings," American Economic Review, LXVI (1976), 430-35

Dickens, Wilham T , and Lawrence F Katz, "Inter-Industry Wage Differences and Industry Characteristics," in Kevin Lang and Jonathan S Leonard, eds, Unemployment and the Structure of Labor Markets (New York, NY Basil Blackwell, 1987a)

Dickens, Willam T, and Lawrence F Katz, "Interındustry Wage Differences and Theories of Wage Determination," National Bureau of Economic Research Working Paper No 2271, 1987b

Duncan, Otıs D , "Ability and Achievement," Eugentcs Quarterly, XV (1968), 1-11

Freeman, Richard, "Longitudinal Analyses of the Effects of Trade Unions," Journal of Labor Economics, II (1984), 1-26 
Grbbons, Robert, and Lawrence Katz, "Does Unmeasured Abllty Explain InterIndustry Wage Differences?" Review of Economic Studies, LIX (1992), 515-35

Griliches, Zv, "Wages of Very Young Men," Journal of Political Economy, LXXXV, Part 2 (1976), S69-85

"Estimating the Returns to Schooling Some Econometric Problems," Econometrica, XLV (1977), 1-22

"Stbling Models and Data in Economics Beginnings of a Survey," Journal of Polttical Economy, LXXXVII, Part 2 (1979), S37-64

Griliches, Zvı, Bronwyn Hall, and Jerry Hausman, "Missing Data and Self-Selection in Large Panels," Annales de L'INSEE, XXX-XXXI (1978), 137-76

Griliches, Zvi, and William Mason, "Education, Income and Ability," Journal of Polttical Economy, LXXX, Part 2 (1972), S74-103

Hauser, Robert M, and Thomas N Daymont, "Schoolıng, Ability and Earnings, Cross-Sectional Findıngs 8 to 14 Years after High School Graduation," Sociology of Education, L (1977), 182-206

Joreskog, Karl G, and Dag Sorbom, LISREL VI Analysts of Linear Structural Relationships by the Method of Maximum Likelihood (Chtcago, IL National Resources, 1984)

Katz, Lawrence F, "Efficiency Wage Theories A Partıal Evaluation," NBER Macroeconomics Annual (1986), 235-76

Krueger, Alan B , and Lawrence H Summers, "Reflections on the Inter-Industry Wage Structure," in Kevin Lang and Jonathan S Leonard, eds, Unemployment and the Structure of Labor Markets (New York, NY Basil Blackwell, 1987)

Krueger, Alan B, and Lawrence H Summers, "Efficiency Wages and the InterIndustry Wage Structure," Econometrica, LVI (1988), 259-94

Lelbowitz, Arleen, "Family Background and Economic Success A Review of the Evidence," in Paul Taubman, ed ,Kinometrics Determinants of Socioeconomic Success Within and Between Familes (Amsterdam North-Holland Publishing Company, 1977)

Murphy, Kevin M , and Robert H Topel, "Efficiency Wages Reconsidered Theory and Evidence," mimeograph, 1987a

Murphy, Kevm M, and Robert H Topel, "Unemployment, Risk, and Earnings Testing for Equalızing Wage Differences in the Labor Market," in Kevin Lang and Jonathan S Leonard, eds, Unemployment and the Structure of Labor Markets (New York, NY Basıl Blackwell, 1987b)

Sewell, William H, and Robert M Hauser, Educatıon, Occupation and Earnıngs Achlevement in the Early Career (New York, NY Academic Press, 1975)

Taubman, Paul, "The Determinants of Earnings Genetics, Family, and Other Environments A Study of White Male Twins," American Economic Review, LXVI (1976), 858-70

Kinometrics Determinants of Socioeconomic Success Within and Between Famlles (Amsterdam North-Holland Publishing Company, 1977) 